\title{
The Isolation and morphology of Some New Bacteriophages Specific for Bacillus and Acetobacter species
}

\author{
BY D. E. BRADLEY \\ Department of Zoology, University of Edinburgh, West Mains Road, \\ Edinburgh 9, Scotland
}

(Received 31 May 1965)

\begin{abstract}
SUMMARY
The best natural habitat for bacteriophages is probably a semi-solid medium containing actively dividing host bacteria. Such conditions are provided for Bacillus and Acetobacter species in rotting grass and apples, respectively. The Bacillus phages found included one with a large head and a contractile tail, and also a so-called killer particle, which had a $350 \AA$ head and a long contractile tail. This particle had the property of killing but not multiplying within a sensitive cell. A new morphological type of virulent Bacillus phage was also isolated; its head was oblong and the tail consisted of a short needle and a plate. The one Acetobacter phage found resembled coliphage T 3 but was of particular interest because of the prominence of the head capsomeres and the three-pronged tail.
\end{abstract}

\section{INTRODUCTION}

Whether by accident or design, the emphasis in phage research has been placed on phages specific to Escherichia coli and other members of the Enterobacteriaceae. Recently, however, the obvious structural, taxonomic and physiological importance of studying a large number of phages for a wide range of host bacteria has led to their examination in the electron microscope by many workers. These results have been reviewed elsewhere (Bradley, 1965a), and it must now be asked whether it is of value to continue to study phages in the electron microscope on this basis. It seems logical to limit descriptions to new morphological types, or phages of particular chemical or physiological interest such as those specific to unusual host bacteria. It is also considered important to study those isolates from natural habitats on hosts not belonging to the Enterobacteriaceae. The genus Bacillus is particularly widespread, and is chosen because its bacteriophages have been somewhat neglected in spite of the fact that they are plentiful in natural habitats. The best natural habitat for a phage is where its host is actively dividing, preferably in a semi-solid medium, for micro-ecological reasons (Bradley, 1965a). Thus, while Bacillus phages are available in the soil, a better habitat would be decomposing vegetation, and this has proved to be the case, in the present work, with rotting lawn mowings.

The usual method for isolating phages consists of plating a suitable laboratory strain of host organism with a cleaned extract of habitat material. Better results can be obtained by isolating the bacteria from the habitat material and using them as potential phage hosts. In the present paper, the examination in this way of rotting lawn mowings and apples is described. In the apples, acid conditions provide 
a microflora dominated by yeasts, Acetobacter, and perhaps Micrococcus species. The electron microscope shows that the phages isolated from both sources are of particular morphological and physiological interest; infectivity tests revealed that one of them was a killer particle (Seaman, Tarmy \& Marmur, 1964).

\section{METHODS}

Plating media and methods. Standard media and procedures were used throughout as follows. The Bacillus species from grass were grown on nutrient broth agar consisting of (g./l.): beef extract, 2 ; yeast extract, 4 ; peptone, $10 ; \mathrm{NaCl}, 10$; agar, $20 ; \mathrm{pH} 7 \cdot 4$. For the double agar layer method of Adams (1959), $1 \%(w / v)$ agar + $1 \%(w / v)$ peptone was used for the soft layer.

Malt extract agar was used for the initial growth of the Acetobacter species (g./l.): malt extract, 17; mycological peptone (Oxoid), 3; agar, 30; pH 5.4. Soft agar layers were made from the same medium but with only $15 \mathrm{~g}$. agar/l. For phage propagation it was found that better results were obtained with nutrient broth (pH $7 \cdot 4)$.

These media without agar were used for isolating bacteria from the two habitats.

Isolation of host bacteria. Samples of rotting grass or apple were shaken with 5-10 ml. of the appropriate liquid medium. A loopful of this mixture was streaked on the appropriate nutrient agar plate and incubated overnight at $30^{\circ}$ or $37^{\circ}$. The colonies obtained were selected according to their morphology and further purified by streaking, before growth on slopes. The use of an acid medium with the rotten apple samples ensured that Acetobacter species and yeasts dominated. Occasionally the dominance of Bacillus species was ensured by heating the extract from the rotting grass at $80^{\circ}$ for 20 min., leaving the Bacillus spores viable. The heated extract was then streaked on plates.

Isolation, purification, and growth of phages. The extract of habitat material obtained as described was centrifuged at $2000 \mathrm{~g}$ for $10 \mathrm{~min}$. to remove bacteria; sometimes an extract was shaken with a little chloroform before centrifugation to help to decrease viable bacteria. (A residue of chloroform can inhibit bacterial growth in subsequent procedures.) A sample (0.3 ml.) of this extract was plated out by the double agar layer method (Adams, 1959) with each of the bacterial isolates obtained above. Phages formed plaques after incubation overnight. These plaques were picked with a platinum loop and suspended in about $1 \mathrm{ml}$. appropriate liquid medium. Purification was achieved by several cycles of plating and plaque picking.

In the case of the Acetobacter phage (AA-1), no plaques were seen when an extract of rotting apple was plated with host bacteria. To ensure that plaques were not missed because of bacterial overgrowth, the plate was extracted with liquid medium, the extract centrifuged to deposit bacteria, and then tested for activity against the original host by the spot test. A positive result indicated the presence of a phage; it was grown to high titre as described below.

High-titre phage preparations for electron microscopy were obtained by confluent lysis of host bacteria on 6 in. plates and extracting the phage with neutral $0 \cdot 1 \mathrm{M}$-ammonium acetate. Then extracts were cleaned by alternate cycles of centrifugation at $2000 \mathrm{~g}$ for $15 \mathrm{~min}$., and at 10,000-15,000 $\mathrm{g}$ for $4 \mathrm{hr}$ (angle head). The final pellet was re-suspended in $0.1-0.2 \mathrm{ml}$. neutral $0.1 \mathrm{M}$-ammonium acetate per plate extracted. 
A mixture of the dominant phages in a sample of habitat material was obtained by plating $1 \mathrm{ml}$. liquid medium extract by the double agar layer method. Extraction of the plate with $0 \cdot 1 \mathrm{M}$-ammonium acetate followed by cycles of centrifugation as above produced a phage suspension suitable for electron microscopy.

Suspensions of killer particles for electron microscopy were prepared by extracting a double agar layer plate of host bacterium with about $3 \mathrm{ml} .0 \cdot 1 \mathrm{M}$-ammonium acetate; this extract contained a high concentration of spontaneously released particles. The general appearance in the electron microscope suggested that about $10^{8}$ particles were extracted from each 3 in. diameter plate.

The spot test for phage activity. This was done as previously described (Bradley, 1964) by placing a drop of phage suspension on a double agar layer plate of host bacteria and observing clearing or otherwise after incubation. The test was also used to distinguish killer particles from virulent phages as follows. Both killer particles and virulent phages give cleared areas with the spot test at high concentrations. However, when a series of dilutions is spotted on a plate, the cleared area will become uniformly more opaque as the dilution is increased. With a virulent phage the clear area will remain clear until with increasing dilution it will become broken into a series of discrete plaques. But a killer particle suspension giving a positive spot test will form no plaques when plated out in serial dilutions. The reason for this is obvious : a killer particle destroys one host cell, but a multiplying virulent phage many. The killer particle's 'plaque' will thus consist of the area occupied by one bacterium, and be invisible. If there are enough killer particles in a small area of plate, they will destroy all the bacteria, creating a clear area, and thus showing a positive spot test.

Table 1. Numbers of phages and hosts

$\begin{array}{ccc}\text { Phage no. } & \text { Host bacterium species } & \text { Host strain no. } \\ \text { GA-1 } & \text { Bacillus subtilis } & \text { G } 1 \text { R } \\ \text { GA-2 } & \text { B. subtilis } & \text { G } 1 \text { R } \\ \text { GF-2 } & \text { B. subtilis } & \text { G6 } \\ \text { AA-1 } & \text { Acetobacter } \text { sp. } & \text { A2 }\end{array}$

The release of killer particles or temperate phages from a bacterium can be detected by spotting a suspension of the suspected bacterium on a double layer plate of the supposedly sensitive host. After incubation, the surface colony will be surrounded by a clear border in the soft agar if it releases particles active against the bacterium under test. This was used to demonstrate the similarity of the killer particle GA-2 to a temperate phage.

Electron microscopy. The standard negative staining procedure (Brenner \& Horne, 1959) with neutral $2 \%(\mathrm{w} / \mathrm{v})$ potassium phosphotungstate was used throughout.

Identification and numbering of bacteria and phages. Members of the genus Bacillus were identified at species level and those of Acetobacter at the generic level by standard tests done at the Department of Agriculture, University of Edinburgh, and in the case of Bacillus spp. also by spore morphology (Bradley \& Franklin, 1958). To avoid confusion arbitrary laboratory numbers are quoted as well (see Table 1). Arbitrary numbers were also given to phage isolates in the absence of any recognized system of nomenclature. 


\section{RESULTS \\ Bacillus subtilis phage $G A-1$}

This new morphological type is shown in Pl. 1, figs. 1-6. It has an oblong head with a six-sided outline of length $570 \AA$ and width about $400 \AA$. The short tail is a tube $60 \AA$ wide and $350 \AA$ long. Part of the way down there is a plate-like structure attached to the base of the head by fibrous material. The plate has several downwardpointing prongs ( $\mathrm{Pl} .1$, figs. 4, 5); three are most often visible, but some particles (see the one at the bottom left in $\mathrm{Pl}$. 1, fig. 1) appear to have four. The prongs are about $70 \AA$ long and are attached to prominent subunits on the plate. The micrographs also illustrate some other points. In Pl. 1, fig. 1, the top particle has lost part of its contents; head capsomeres can just be seen, but not in a clear arrangement. Another particle has lost its tail and shows a flattening at the point of attachment of the tail. This is also shown particularly clearly in Pl. 1, fig. 2, the particle here being completely full; the micrograph also shows the fibrous material between the plate and the head. In Pl. 1, fig. 3, fibrous material is again visible together with the point of attachment between the tail and head, where there is a plug of protein, commonly found in all tailed phages. A comparison of the tails in the empty or partly empty virions in Pl. 1, figs. 1, 3, with the full one in Pl. 1, fig. 2, shows that the former are hollow and the latter solid. This feature has been observed particularly in phages with long non-contractile tails (Bradley \& Kay, 1960). In Pl. 1, fig. 6, two particles are shown attached to a piece of debris; this suggests that adsorption takes place tail-first in the conventional manner.

\section{Bacillus killer particle $G A-2$}

An intact killer particle GA-2 is shown in Pl. 1, fig. 8; it consists of a small head with a massive tail. The head is $350 \AA$ in diameter, but the tail is $2100 \AA$ long and has the conventional appearance of a contractile type with a striated sheath and tail fibres. The number of tail fibres is difficult to ascertain; 4 or 5 is most likely. An empty head and short length of tail core are shown in Pl. 1, fig. 7. The core is in the form of a hollow tube about $90 \AA$ in diameter and there is a lump of protein at the attachment to the head. The head membrane is continuous and does not show any obvious capsomeres. The particle in Pl. 2, fig. 9, has a partly empty head and shows a regular hexagonal outline. The tail capsomeres are clearer on the headless tail (Pl. 2, fig. 10) and they are undoubtedly tubular in form (end-on capsomeres arrowed) and are probably arranged in screw symmetry. Plate 2, fig. 11, shows a virion with a contracted tail, though the head has remained full. Here, the top of the sheath is adjacent to the head, only separated from it by a small collar, which is not obvious in the other micrographs so far mentioned. It appears from Pl. 2, figs. 12, 13, and other micrographs, that the contracted sheath can take up any position on the core. This sheath also shows two dark lines running down its length; these are clearer in Pl. 2, fig. 13. A headless tail with contracted sheath is shown in $\mathrm{Pl} .2$, fig. 14; two small discs can be seen at the top of the core where it would be joined to the head. These probably represent a 'double washer' arrangement by which the massive tail is firmly attached to the head; this feature has only previously been found on two phages, one specific to Escherichia coli (Bradley, 1964) and one to Pseudomonas syringae (Matthews \& Bradley, 1964). 
In $\mathrm{Pl} .2$, fig. 15, the major portion of a tail core with the head still attached is shown. The head protein seems to have been slightly degraded, revealing the 'double washer' arrangement clearly. It would appear from this micrograph that the protein plug in Pl. 1, fig. 7, is in fact the inner 'washer'.

The use of the spot test and other plating methods described above indicated that killer particle GA-2 was able only to kill sensitive host bacteria and not to multiply within them. Particles of this morphology were found in large numbers in extracts of plates of Bacillus subtilis and $B$. licheniformis, presumably being spontaneously released as is the case with most temperate phages; there was no need to induce them with ultraviolet radiation, antibiotics or hydrogen peroxide. Other particles of varied morphology (Pl. 2, fig. 16) were found similarly associated with the Bacillus species isolated from rotting grass, but were not examined in detail.

\section{Bacillus subtilis phage GF-2}

Several phage isolates with a conventional contractile tail were found associated with another strain of Bacillus subtilis; one of them (GF-2) was retained. A micrograph of an intact virion is shown in Pl. 3, fig. 17. The head is hexagonal and about $1150 \AA$ in mean diameter. The rather thin tail is $2800 \AA$ long and has a base-plate but no obvious tail fibres or pins. A head is shown in Pl. 3, fig. 20, which looks too complex to be an octahedron but might well be an icosahedron. The tail can contract in two ways: it either decreases its length by one half (Pl. 3, fig. 18), a state of affairs observed after storage for some months, or by about one quarter (Pl. 3, fig. 19). The latter state occurred spontaneously on the preparation of a fresh lysate. The amount of contraction associated with adsorption is not known for certain, but is probably that shown in $\mathrm{Pl}$. 3, fig. 18, resembling other contractile phages.

\section{Acetobacter phage $A A-1$}

The unusual morphology of this Acetobacter phage is shown in Pl. 3, fig. 21, at low magnification; it is not unlike coliphage T3, but differs in the tail structure, which consists of three prongs when seen in profile. Also the head capsomeres are clearly visible though it is not possible to see how they are arranged. The capsomeres and the hexagonal outline of the head are clearer in Pl. 3, fig. 22. The head size is about $650 \AA$, near to that of coliphage T 3. The tail is shown better in Pl. 3, fig. 23. It is not unlike the base-plate of T-even coliphages, but is smaller, the total length being only $185 \AA$, and the prongs $130 \AA$. The head capsomeres in this figure show as apparent breaks in the protein coat with a spacing of about $130 \AA$. In the empty virion in Pl. 3, fig. 24, a different picture is revealed. There are only two tail prongs, and the head capsomeres appear as hollow circular units at a spacing of only $45 \AA$. However, the coarse $130 \AA$ structure is again apparent in the capsids in Pl. 3, fig. 25. These different appearances present a problem in interpretation which will be discussed below. A contaminant phage was also found in a preparation of Acetobacter phage AA-1; this is seen in Pl. 3, fig. 26, and shows that other morphological types may be found in rotting apples. This contaminant resembles some Staphylococcus phages (Bradley, 1963a) and so might be presumed to come from a related genus, perhaps $M$ icrococcus. 


\section{DISCUSSION}

The virulent Bacillus subtilis phages. The new morphological type, Bacillus phage GA-1, which appears to be common in nature, is the most important of the two described here. It belongs to the morphological group of phages with short noncontractile tails (Bradley, 1965a) and is closest to coliphage P22 (Anderson, 1960). Although one cannot be certain from the electron micrographs shown about the true shape of phage GA-1, by assuming that the rules of symmetry are obeyed (Bradley, 1963a, $b$ ) certain deductions can be made. To ascertain the head shape, the radial symmetry of the tail must first be determined. The micrographs show a tail plate with three or four visible pins or subunits, and this strongly suggests a sixfold rather than a fourfold or fivefold radial symmetry. The base-plate of T-even coliphages, with its six pins; usually shows only three in profile in an electron micrograph, a similar state of affairs. Thus, with the tail probably having a sixfold radial symmetry, the long axis of the head to which it is attached will be the same. This, when considered in conjunction with the outline of the head perpendicular to the long axis, as seen in electron micrographs, suggests a bipyramidal hexagonal prism. It is to be noted that the $T$-even coliphage head was thought to be this shape for what were basically the same reasons, but facets visible on electron

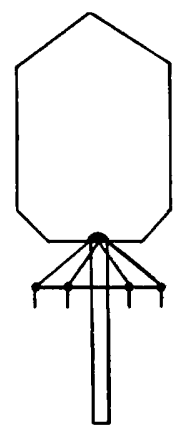

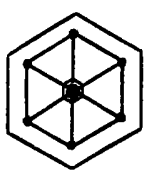

Fig. 1. Diagram of Bacillus phage GA-1; $(a)$ side view;

(b) view down axis of tail, $\times \mathbf{3 5 0 , 0 0 0}$.

micrographs showed the assumption to be incorrect (Bradley, 1965 b). No facets can be seen here, but the head outline is quite different to that of the T-even coliphages, which are much fatter-i.e. they have a larger width/length ratio. It seems not unlikely that Bacillus phage GA-1, with its more slender appearance, shows the true outline of a bipyramidal hexagonal prism. The Staphylococcus phage 594 $\mathrm{n}$ (Bradley, 1963a) has a similarly shaped head. Unfortunately it is likely to be very difficult to demonstrate a bipyramidal hexagonal prism conclusively in the electron microscope. These interpretations are illustrated diagrammatically in Fig. 1. Bacillus phage GF-2 is described here because it again raises the question of head shape. Phages with heads more than $400 \AA$ in size seem to prefer the octahedral form, though there are exceptions. One of these (SP 8), specific to Bacillus subtilis, was described by Davison (1963); it resembles Bacillus phage GF-2 though the tail is much shorter; convincing evidence is shown to indicate that SP8 is an icosa- 
hedron. A similar isolate (SP 50) was described by Eiserling \& Boy de la Tour (1965), who stated that this phage head is also probably an icosahedron, though their micrographs suggest that an octahedron is more likely. However, the tail plate of SP 50 has five distinct pins, which also appears to be the case with SP 8. Thus, in the case of SP 8 the symmetry rule is obeyed, and there is no reason to suppose that SP 50 is an exception. With Bacillus phage GF-2, there are no tail pins to act as a guide to head shape, but from Pl. 3, fig. 20, and the two precedents it also seems likely to have an icosahedral head. From these observations it is important to note that an electron micrograph of an icosahedral body can often appear very like one of an octahedral body; care needs to be taken in the interpretation (Bradley, $1963 b)$.

These Bacillus phages are thus further examples of virions which conform to the symmetry rule. It is becoming increasingly necessary to establish the extent to which this rule is obeyed since the validity of various recent important interpretations of the T-even coliphage head shape may depend upon it (Bradley, 1965 $b$; Moody, 1965; Boy de la Tour \& Kellenberger, 1965).

Bacillus killer particles. There have been several reports of the isolation of killer particles from strains of Bacillus subtilis and $\boldsymbol{B}$. licheniformis, most notably by Seaman et al. (1964) and Stickler, Tucker \& Kay (1965). These authors reported that the particles were only obtained after induction of the host strain with mitomycin $\mathrm{C}$ and with hydrogen peroxide, respectively. The particle GA-2, on the other hand, was released spontaneously from both these species under normal growth conditions. Perhaps because of its unusual appearance, the GA-2 morphological form was studied in greatest detail by the above authors. The electron micrographs of Stickler et al. (1965) give an opportunity for comparison, and the only real differences appear to be in the length of the tail, and hence the number of striations. According to Stickler et al. (1965) there were six tail fibres, but, from the observations given here, five would appear more likely. If the symmetry rule holds, this would conveniently fit the fivefold axis of an icosahedral head, a form favoured by small viral capsids. The tail capsomeres are particularly interesting. They appear circular in some places (Pl. 2, fig. 10, arrowed), indicating that they are tubular. The results of acridine orange staining (Bradley, $1965 \mathrm{c}$ ) suggest that the head contains doublestranded DNA like the other killer particles mentioned.

Acetobacter phage $\boldsymbol{A A - 1}$. It is difficult to obtain electron micrographs which clearly demonstrate the shape of this phage but again, assuming the symmetry rule, a probable form can be deduced from the tail. The appearance of three or two prongs strongly indicates a fourfold radial symmetry, which would make the head octahedral. The number of visible prongs would depend upon the orientation of a square plate having one at each corner. Judging from the apparent size of the head capsomeres in $\mathrm{Pl}$. 3, figs. 21-23, one would expect to see their arrangement clearly, but the empty virion seems to have much smaller ones. It is considered that the large capsomeres are really an artifact produced by the superposition of the regular array of smaller ones on each side of the head; it must be remembered in interpreting electron micrographs of negatively stained virions that contrast is produced by portions of the viral surface adjacent to as well as away from the specimen support film.

Conclusion. It seems likely from the foregoing that the examination of bacterio- 
phages and similar particles as they occur in nature is likely to produce interesting material for physiological, chemical and genetic studies. The Bacillus killer particles described appear to lie somewhere between the non-infective virus-like rods of Saprospira grandis (Correll \& Lewin, 1964) and a true temperate phage. It is interesting to list the various particles found associated with bacteria. There are virulent phages with ability to multiply within the host and temperate phages, many of which behave in a virulent fashion towards sensitive hosts. The killer particles are produced from the host in the same way as temperate phages, but are unable to act in a virulent fashion, or, in the case of GA-2, to lysogenize a sensitive host. The bacteriocins resemble the killer particles in that they cannot multiply. When one compares the properties of bacteriocins with those of the killer particles one finds such a close resemblance that it seems possible that many if not all of those so far discovered are defective phages. This is contrary to the views expressed by Jacob \& Wollman in 1959, but killer particles had not at that time been discovered. Further evidence to support this has been provided by Sandoval, Reilly \& Tandler (1965), who showed that colicin 15 is probably a killer particle. Finally there are the non-infective rods of Saprospira grandis, again released spontaneously like Bacillus killer particle GA-2. Each of the members in this list is linked in some physiological characteristic and the continued study of their interrelationships may provide information on the question of the origin of bacteriophages and hence of viruses in general.

The author would like to thank Drs T. Gibson and R. Whittenbury (Edinburgh University Department of Agriculture) for identifying the bacteria used, and Drs D. J. Stickler, R. G. Tucker and D. Kay (Oxford University) and Drs F. A. Eiserling and E. Boy de la Tour (Geneva University) for kindly providing information and manuscripts before publication. The author is also particularly grateful to Miss C. A. Dewar for indispensable technical assistance.

\section{REFERENCES}

Adams, M. H. (1959). Bacteriophages. New York and London: Interscience Publishers.

Anderson, T. F. (1960). On the fine structure of the temperate bacteriophages P 1, P2 and P 22. Proc. Eur. Reg. Conf. electron Microscopy, Delft, 2, 1008.

Boy de la Tour, E. \& Kellenberger, E. (1965). Aberrant forms of T-even phage head. Virology (in the Press).

Bradeley, D. E. (1963a). The structure of some Staphylococcus and Pseudomonas phages. J. Ultrastruct. Res. 8, 552.

Bradley, D. E. (1963b). The structure of coliphages. J. gen. Microbiol. $31,435$.

Bradley, D. E. (1964). The structure of some bacteriophages associated with male strains of Escherichia coli. J. gen. Microbiol. 35, 471.

Bradley, D. E. $(1965 a)$. The morphology and physiology of bacteriophages as revealed by the electron microscope. J. R. microsc. Soc. (in the Press).

Bradley, D. E. $(1965 b)$. The structure of the head, collar and base-plate of 'T-even' type bacteriophages. J. gen. Microbiol. 38, 395.

BRADLEY, D. E. $(1965 c)$. Staining of bacteriophage nucleic acids with acridine orange. Nature, Lond. 205, 1230.

Bradley, D. E. \& Franklin, J. G. (1958). Electron microscope survey of the surface configuration of spores of the genus Bacillus. J. Bact. 76, 618.

Bradiey, D. E. \& KAY, D. (1960). The fine structure of bacteriophages. J. gen. Microbiol. 23, 553. 

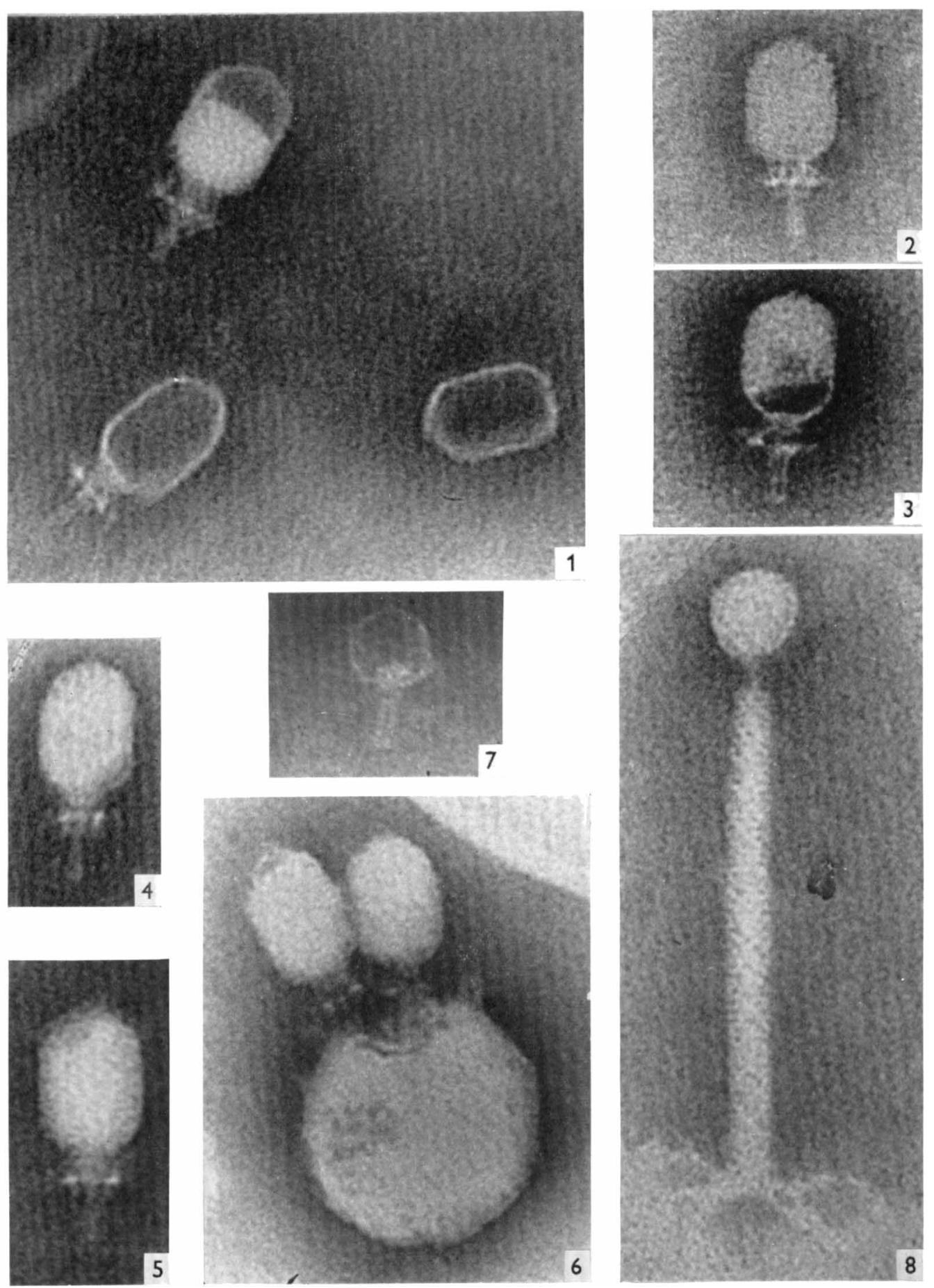

D. E. BRADLEY 

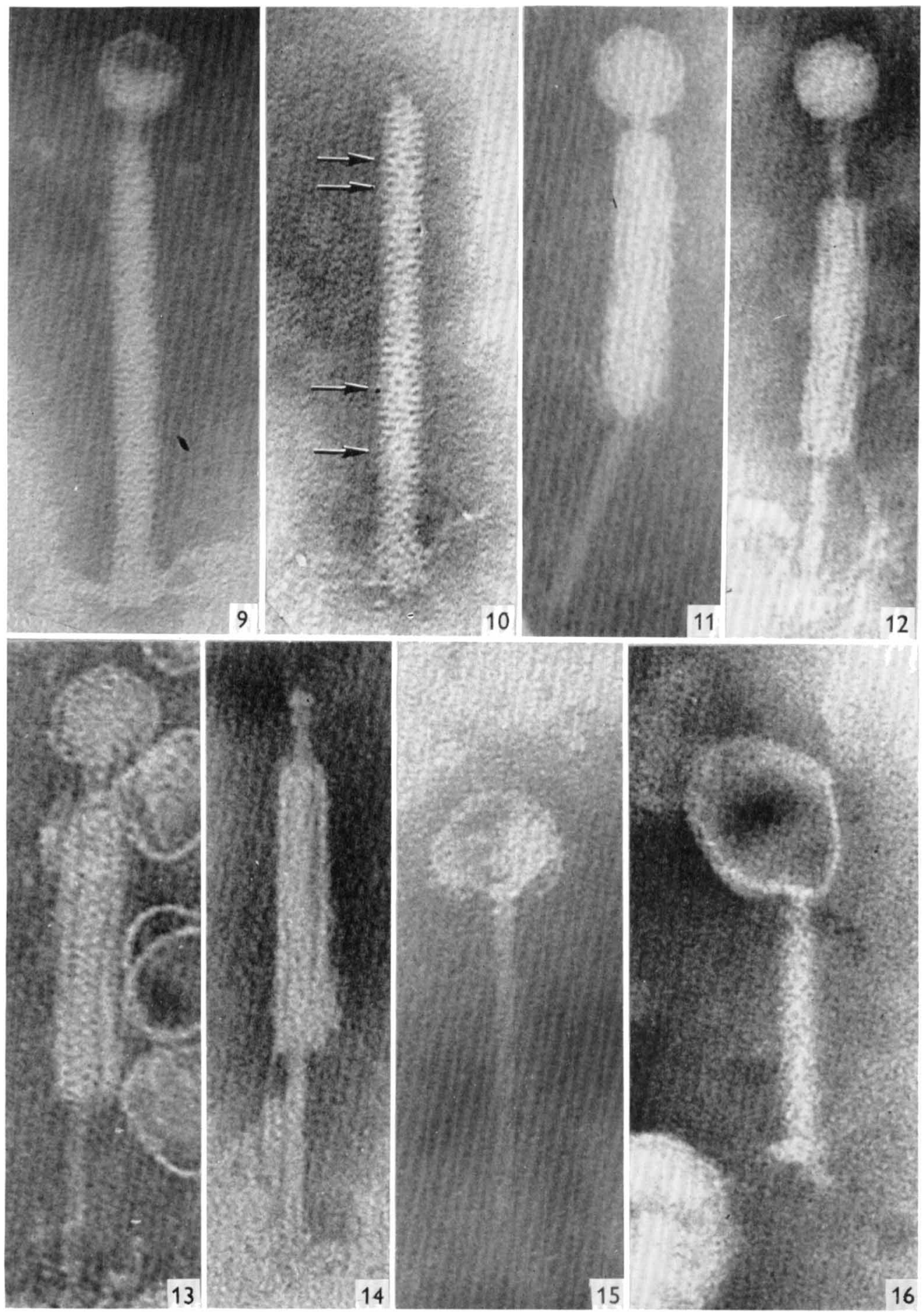

D. E. BRADLEY 

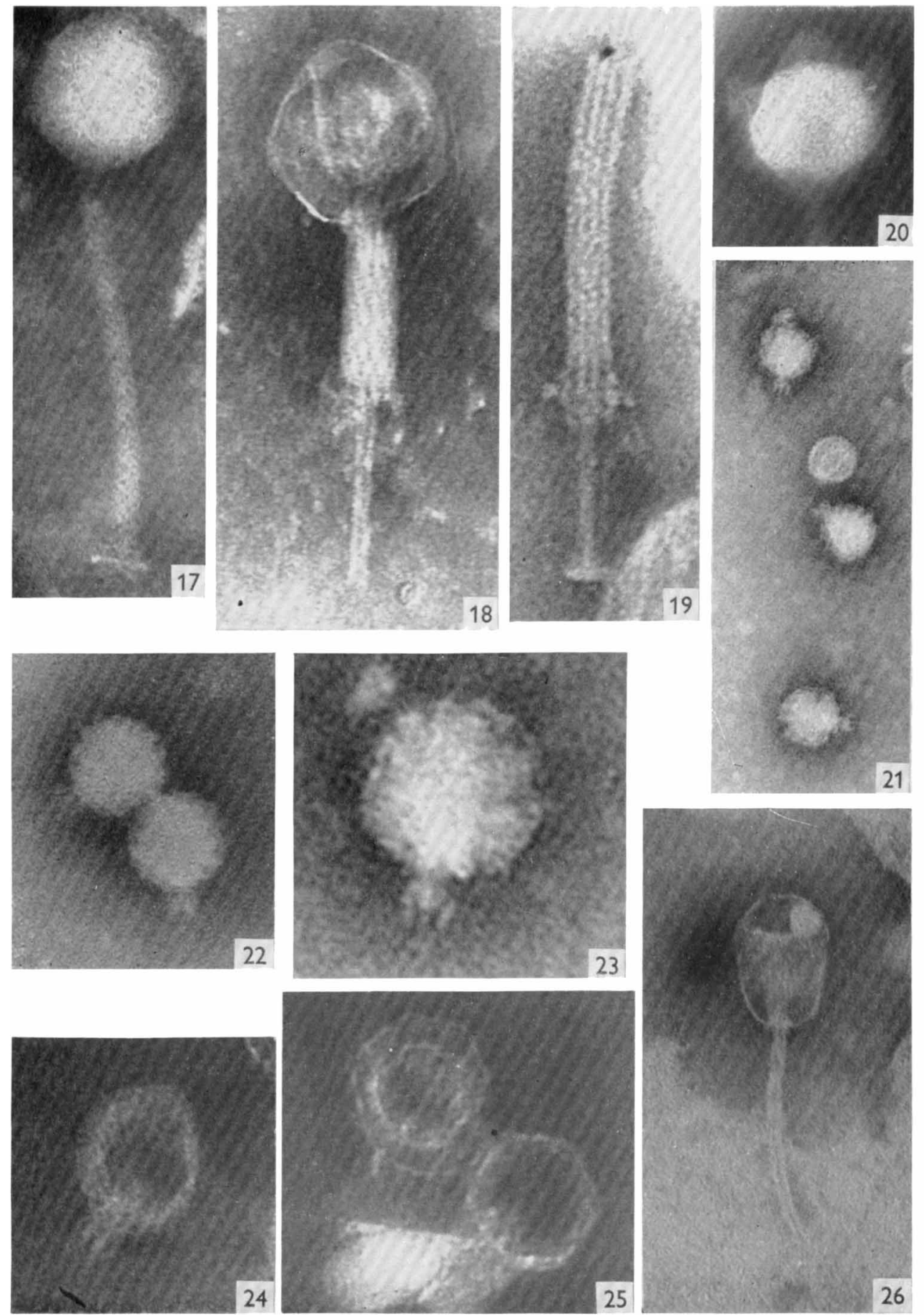

D. E. BRADLEY 

Brenner, S. \& Horne, R. W. (1959). A negative staining method for high resolution electron microscopy of viruses. Biochim. biophys. Acta, 34, 103.

Correll, D. L. \& Lewin, R. A. (1964). Rod-shaped ribonucleoprotein particles from Saprospira. Can. J. Microbiol. 10, 63.

Davison, P. F. (1963). The structure of bacteriophage SP 8. Virology, 21, 146.

Eiserling, F. A. \& BoY de LA Tour, E. (1965). Capsomeres and other structures observed on some bacteriophages. Pathologia Microbiol. 28, 175.

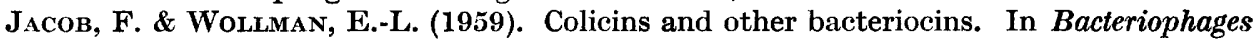
by M. H. Adams. London and New York: Interscience Publishers.

Matthews, M. A. \& Bradley, D. E. (1964). Preliminary observations on two new bacteriophages from Pseudomonas and Serratia. Proc. Eur. Reg. Conf. electron Microscopy, Prague, vol. B, p. 543.

Moody, M. F. (1965). Virology (in the Press).

Sandoval, H. K., Reilly, C. H. \& Tandler, B. (1965). Colicin 15 : possibly a defective bacteriophage. Nature, Lond. 205, 522.

Seaman, E., Tarmy, E. \& Marmur, J. (1964). Inducible phages of Bacillus subtilis. Biochemistry, 3, 607 .

Stickler, D. J., Tucker, R. G. \& KAY, D. (1965). Bacteriophage-like particles released from Bacillus subtilis after induction with hydrogen peroxide. Virology, 26, 142.

\section{EXPLANATION OF PLATES}

Figs. 1-5. Phage GA-1, $\times 333,000$.

Plate 1

Fig. 6. Phage GA-1 particles adsorbed to debris, $\times 333,000$.

Fig. 7. Empty head and part of tail core of killer particle GA-2, $\times 333,000$.

Fig. 8. Intact killer particle GA-2, $\times 333,000$.

Plate 2

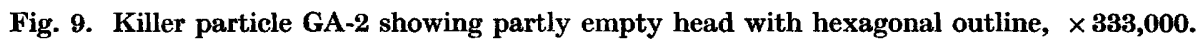

Fig. 10. Headless tail of GA-2 showing tail capsomeres, $\times 333,000$.

Figs. 11-13. GA-2 killer particles with contracted tail sheaths, $\times 333,000$.

Fig. 14. Headless GA-2 particle with double washer, $\times \mathbf{3 3 3 , 0 0 0}$.

Fig. 15. Sheathless GA-2 particle showing core and double washer, $\times \mathbf{3 3 3 , 0 0 0}$.

Fig. 16. Another morphological type of killer particle, $\times 333,000$.

Fig. 17. Intact GF-2 virion, $\times 200,000$.

Plate 3

Fig. 18. GF-2 with fully contracted sheath, $\times 200,000$.

Fig. 19. Partly contracted GF-2 sheath, $\times 333,000$.

Fig. 20. GF-2 head showing numerous facets, $\times 200,000$.

Fig. 21. Intact virions of phage AA-1, $\times 110,000$.

Fig. 22. Phage AA-1 virions showing head capsomeres, $\times 220,000$.

Fig. 23. Virion of phage AA-1 showing three-pronged tail, $\times 380,000$.

Fig. 24. Empty virion of AA-1 with two-pronged tail, $\times 333,000$.

Fig. 25. Empty virions of AA-1, $\times 333,000$.

Fig. 26. Particle of a phage from rotten apple, $\times 200,000$. 